Pacific

Journal of

Mathematics

\title{
UNCOUNTABLY MANY INEQUIVALENT LIPSCHITZ HOMOGENEOUS CANTOR SETS IN $\mathbb{R}^{3}$
}

DENNIS GARITY, DUŠAN REPOVŠ AND MATJAŽ ŽELJKo 


\title{
UNCOUNTABLY MANY INEQUIVALENT LIPSCHITZ HOMOGENEOUS CANTOR SETS IN $\mathbb{R}^{3}$
}

\author{
DENNIS GARITY, DUŠAN REPOVŠ AND MATJAŽ ŽELJKO
}

\begin{abstract}
General techniques are developed for constructing Lipschitz homogeneous wild Cantor sets in $\mathbb{R}^{3}$. These techniques, along with Kauffman's version of the Jones polynomial and previous results on Antoine Cantor sets, are used to construct uncountably many topologically inequivalent such wild Cantor sets in $\mathbb{R}^{3}$. This use of three-dimensional finite link invariants to detect distinctness among wild Cantor sets is unexpected. These Cantor sets have the same Antoine graphs and are Lipschitz homogeneous. As a corollary, there are uncountably many topologically inequivalent Cantor sets with the same Antoine graph.
\end{abstract}

\section{Introduction}

Malešič and Repovš [1999] have constructed a specific example of a wild Cantor set in $\mathbb{R}^{3}$ that is Lipschitz homogeneously embedded. This answered negatively a question in [Repovš et al. 1996] as to whether Lipschitz homogeneity of a Cantor set implied tameness. In this paper, we introduce more general techniques for detecting the Lipschitz homogeneity of Cantor sets in $\mathbb{R}^{n}$. These techniques allow us to construct uncountably many topologically distinct Lipschitz homogeneous wild Cantor sets in $\mathbb{R}^{3}$. These Cantor sets are all simple Antoine Cantor sets with the same Antoine graph as defined in [Wright 1986]. The fact that the constructed Cantor sets are all topologically distinct is a consequence of a result of Sher [1968] and a computation of Kauffman's version [1988] of the Jones polynomial for the center lines of certain tori used in the construction. It is hoped that the techniques in this paper may also prove to be applicable to showing that certain Blankinship type Cantor sets [Blankinship 1951; Eaton 1973] in $\mathbb{R}^{n}$ for $n \geq 4$ can be constructed so as to be Lipschitz homogeneously embedded.

MSC2000: primary 54E45, 54F65; secondary 57M30, 57N10.

Keywords: wild Cantor set, Lipschitz homogeneity, similitude, coefficient of similarity, defining sequence, link invariant.

Garity was supported in part by NSF grants DMS 0139678 and DMS 0104325. Repovš and Matjaž were supported in part by MESS grant 0101-509. All authors were supported in part by MESS grant SLO-US 2002/01. 


\section{Notation and Background}

Lipschitz maps and similitudes. A map $S: \mathbb{R}^{n} \rightarrow \mathbb{R}^{n}$ is said to be a Lipschitz map if there exists a constant $\lambda$ such that

$$
|S(x)-S(y)| \leq \lambda|x-y| \text { for every } x, y \in \mathbb{R}^{n}
$$

and the smallest such $\lambda$ is called the Lipschitz constant of $S$. In the special case when

$$
|S(x)-S(y)|=\lambda|x-y| \text { for every } x, y \in \mathbb{R}^{n}
$$

the map $S$ is called a similarity and the number $\lambda$ is called the coefficient of similitude. Finally, when $\lambda=1$ the map $S$ is called an isometry.

A Cantor set $C$ in $\mathbb{R}^{3}$ is Lipschitz homogeneously embedded if for each pair of points $x$ and $y$ in $C$ there is a Lipschitz homeomorphism $h: \mathbb{R}^{n} \rightarrow \mathbb{R}^{n}$ with $h^{-1}$ also Lipschitz such that $h(C)=C$ and $h(x)=y$.

Coordinates of points in Cantor sets. In the applications of this section, the compact set $X$ mentioned below will in general be a solid torus.

Let $G_{i}, 1 \leq i \leq M$, be finite index sets and let $\mathbf{S}_{i}=\left\{S_{g}: \mathbb{R}^{n} \rightarrow \mathbb{R}^{n}: g \in G_{i}\right\}$ be a set of similarities having the same coefficient $\lambda_{i}$ of similitude. Let $\mathbf{S}=\cup \mathbf{S}_{i}$. Additionally, suppose that there exists a compact set $X \subset \mathbb{R}^{n}$ such that

(1) $S_{g}(X) \subset \stackrel{\circ}{X}$ for each $g \in G_{i}$ and

(2) the sets $S_{g}(X)$ are pairwise disjoint, $g \in G_{i}$.

Let $\mathbf{T}=\left(n_{1}, n_{2}, \ldots\right)$ be a fixed sequence where each $n_{i}$ is in $\{1, \ldots, M\}$. Let $G^{k}=G_{n_{1}} \times G_{n_{2}} \times \cdots \times G_{n_{k}}, G_{k}^{\infty}=\prod_{i=k}^{\infty} G_{n_{i}}$ and $G^{\infty}=\prod_{i=1}^{\infty} G_{n_{i}}$. For each multiindex $\gamma=\left(g_{1}, g_{2}, \ldots g_{k}\right) \in G^{k}$, write

$$
S_{\gamma}=S_{g_{1}} \circ S_{g_{2}} \circ \ldots \circ S_{g_{k}} \quad \text { and } \quad X_{\gamma}=S_{\gamma}(X) \text {. }
$$

In particular, $X_{g}=S_{g}(X)$ for $g \in G$.

The number of components of a multiindex $\gamma$ is called the depth of $\gamma$. So depth $\gamma=k$ if $\gamma \in G^{k}$.

$$
\text { Let } X_{k}=\bigcup_{\text {depth } \gamma=k} X_{\gamma} .
$$

It is well-known [Hutchinson 1981] that the intersection of the sequence of sets $X \supset X_{1} \supset X_{2} \supset \cdots$ is a Cantor set. Denote this set by $|(\mathbf{S}, \mathbf{T})|$. This Cantor set is self-similar if $\mathbf{T}$ is repeating.

For an infinite multiindex $\gamma=\left(g_{1}, g_{2}, g_{3}, \ldots\right) \in G^{\infty}$ define

$$
\gamma^{k}=\left(g_{1}, g_{2}, \ldots g_{k}\right) \quad \text { and } \quad X_{\gamma}=\bigcap_{k=1}^{\infty} X_{\gamma^{k}} .
$$


Obviously, each $X_{\gamma}$ is a singleton, consisting of a point from the Cantor set $|(\mathbf{S}, \mathbf{T})|$ and for each point from $|(\mathbf{S}, \mathbf{T})|$ there exists exactly one such multiindex $\gamma$. The components of $\gamma$ are called the coordinates of the corresponding point from the Cantor set $|(\mathbf{S}, \mathbf{T})|$.

Finally define a juxtaposition of multiindices as follows. If $\delta=\left(d_{1}, d_{2}, \ldots d_{k}\right)$ is a finite multiindex and $\gamma=\left(g_{1}, g_{2}, \ldots\right)$ is finite or infinite then let

$$
\delta \gamma=\left(d_{1}, d_{2}, \ldots d_{k}, g_{1}, g_{2}, \ldots\right) .
$$

In the special case when depth $\gamma=1$, we have $\gamma=g_{1}$ and $\delta g_{1}=\left(d_{1}, d_{2}, \ldots d_{k}, g_{1}\right)$.

Antoine Cantor sets. We give a brief summary of results from [Sher 1968] and [Wright 1986]. Sher's results are needed in our proof of our main theorem, while Wright's are used in our observation about Antoine graphs associated with the Cantor sets we construct.

An Antoine Cantor set $C$ in $\mathbb{R}^{3}$ is a Cantor set meeting the following conditions.

(1) $C$ has a defining sequence $M_{1}, M_{2}, \ldots$, each $M_{i}$ consisting of the union of a finite number of pairwise disjoint standard unknotted solid tori in $\mathbb{R}^{3}$ and $M_{1}$ consisting of a single solid torus.

(2) The tori in $M_{i}$, for $i \geq 2$, can be listed in a sequence $T_{i, 1}, T_{i, 2}, \ldots, T_{i, n(i)}$ so that $T_{j}$ and $T_{k}$ are of simple linking type if $j-k= \pm 1 \bmod n$ and do not link if $j-k \neq \pm 1 \bmod n$.

(3) The linked chain of tori $T_{i, 1}, T_{i, 2}, \ldots, T_{i, n(i)}$ have winding number greater than 0 in the torus at the previous stage that contains them.

If, in condition (3), the winding number is required to be 1 , and if each $n(i) \geq 4$, we call the resulting Cantor set a simple Antoine Cantor set. Most Antoine Cantor sets in the literature, including the original one [Antoine 1920] are simple.

Sher [1968] showed that if two Antoine Cantor sets $C_{1}$ and $C_{2}$ with defining sequences $M_{1}, M_{2}, \ldots$ and $N_{1}, N_{2}, \ldots$ are equivalently embedded in $\mathbb{R}^{3}$, then there is a homeomorphism $h$ of $\mathbb{R}^{3}$ to itself such that for each $i, h$ takes the tori in $M_{i}$ homeomorphically onto the tori in $N_{i}$. As a consequence, if it can be shown that for some $i$, no such homeomorphism exists, the two Cantor sets are inequivalently embedded. This is the result we will need to construct the uncountably many inequivalently embedded Cantor sets.

Wright [1986] associates an Antoine graph $\Gamma(C)$ with a Antoine Cantor $C$ with defining sequence $M_{1}, M_{2}, \ldots$. The graph $\Gamma$ is a countable union of nested subgraphs $\Gamma_{0} \subset \Gamma_{1} \subset \Gamma_{2} \subset \cdots$. The subgraph $\Gamma_{0}$ is a single vertex. For each vertex $v$ of $\Gamma_{i}-\Gamma_{i-1}$, there is a polygonal simple closed curve with at least 4 vertices $P(v)$ contained in $\Gamma_{i+1}-\Gamma_{i}$ so that if $v$ and $w$ are distinct vertices of $\Gamma_{i}-\Gamma_{i-1}$, then $P(v)$ and $P(w)$ are disjoint. $\Gamma_{i+1}$ consists of $\Gamma_{i}$, the union of the $P(v)$ for $v$ 
in $\Gamma_{i}$, and the union of edges running from $v$ to the vertices of $P(v)$. The vertices of $\Gamma_{i}-\Gamma_{i-1}$ correspond to the components of $M_{i}$.

If $v$ is a vertex of $\Gamma_{i}-\Gamma_{i-1}$ corresponding to a component $C$ of $M_{i}$, then $P(v)$ contains precisely the vertices corresponding to the components of $M_{i+1}$ contained in $C$, and two such vertices are joined by an edge if and only if the corresponding components of $M_{i+1}$ are linked. Here is a diagram of an Antoine graph similar to that in [Wright 1986, p. 252]:

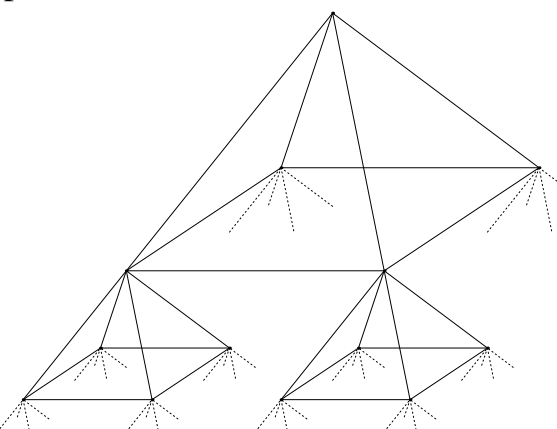

Wright shows that if $C_{1}$ and $C_{2}$ are simple Antoine Cantor sets with different Antoine graphs $\Gamma\left(C_{1}\right)$ and $\Gamma\left(C_{2}\right)$, the Cantor sets are inequivalently embedded.

In our construction, all of the Cantor sets constructed have the same Antoine graph, but are inequivalently embedded.

\section{Constructing Lipschitz homogeneous Cantor sets}

Let $G_{i}, 1 \leq i \leq M, \mathbf{S}_{i}=\left\{S_{g}: \mathbb{R}^{n} \rightarrow \mathbb{R}^{n} ; g \in G_{i}\right\}, X, X_{g}$ and $\mathbf{T}=\left(n_{1}, n_{2}, \ldots\right)$ be as above. The setting to keep in mind when reading Theorem 1 below is that of a simple Antoine Cantor set defined by tori where each stage $m$ torus has $\left|G_{n_{m}}\right|$ stage $m+1$ tori in its interior. For Theorem 1, also assume that each $G_{i}$ is a finite cyclic group, with the group operation written additively.

Theorem 1. For each $i, 1 \leq i \leq M$, suppose that $f_{i}: \mathbb{R}^{n} \rightarrow \mathbb{R}^{n}$ is a Lipschitz homeomorphism and that

(i) $\left.f_{i}\right|_{\mathbb{R}^{n}-\check{X}}=\mathrm{id}$,

(ii) for each $g \in G_{i}$, we have $f_{i}\left(X_{g}\right)=X_{g+1}$ and the diagram

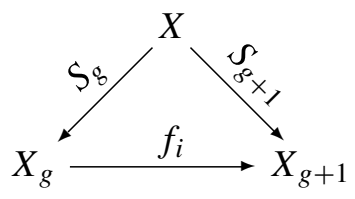

commutes.

Then $|(\mathbf{S}, \mathbf{T})|$ is Lipschitz, homogeneous in $\mathbb{R}^{n}$. 
Proof. The approach to the proof is similar to that used in [Malešič and Repovš 1999, Lemma 1]. The main modification needed is to take into account the presence of more than one finite index set. Fix an arbitrary pair of points $a$ and $b$ in $|(\mathbf{S}, \mathbf{T})|$. We will construct a homeomorphism

$$
h:\left(\mathbb{R}^{n},|(\mathbf{S}, \mathbf{T})|, a\right) \rightarrow\left(\mathbb{R}^{n},|(\mathbf{S}, \mathbf{T})|, b\right)
$$

and prove that both $h$ and $h^{-1}$ are Lipschitz. Let $\alpha=\left(a_{1}, \ldots, a_{k}, \ldots\right)$ and $\beta=$ $\left(b_{1}, \ldots, b_{k}, \ldots\right) \in G^{\infty}$ be the coordinates of $a$ and $b$. For an arbitrary $\gamma=$ $\left(g_{1}, \ldots, g_{k}\right) \in G^{k}$ define the homeomorphism

$$
f_{\gamma}=S_{\gamma} \circ f_{n_{k+1}} \circ S_{\gamma}^{-1}: \mathbb{R}^{n} \rightarrow \mathbb{R}^{n} .
$$

Set

$$
\begin{aligned}
r_{1} & =f_{n_{1}}^{b_{1}-a_{1}}, r_{2}=f_{b_{1}}^{b_{2}-a_{2}}, r_{3}=f_{\left(b_{1}, b_{2}\right)}^{b_{3}-a_{3}}, \ldots, r_{k+1}=f_{\beta^{k}}^{b_{k+1}-a_{k+1}}, \ldots \\
\ell_{i} & =r_{i}^{-1}, \\
h_{k} & =r_{k} \circ r_{k-1} \circ \cdots \circ r_{2} \circ r_{1} .
\end{aligned}
$$

In addition, for notational convenience, let

$$
r_{-1}=r_{0}=\left.i d\right|_{\mathbb{R}^{n}}
$$

It follows by Lemma 2(iv) below that the sequences of homeomorphisms $h_{1}, h_{2}, \ldots$ and $h_{1}^{-1}, h_{2}^{-1}, \ldots$ converge pointwise at all points different from the point $a$ and $b$, respectively. The convergence of the sequences at the point $a$ and at the point $b$ follows from Lemma 2(ii). Denote the limits of the sequences by $h: \mathbb{R}^{n} \longrightarrow \mathbb{R}^{n}$ and $\tilde{h}: \mathbb{R}^{n} \longrightarrow \mathbb{R}^{n}$, respectively. It also follows by Lemma 2 that $h(a)=b$, that $h(|(\mathbf{S}, \mathbf{T})|)=|(\mathbf{S}, \mathbf{T})|$, and that $h \circ \tilde{h}=\tilde{h} \circ h=\operatorname{id}_{\mathbb{R}^{n}}$. It follows from Lemma 3 that $h$ and $\tilde{h}$ are Lipschitz. Thus Theorem 1 is proved.

\section{Lemmas needed for Theorem 1.}

Lemma 1. The homeomorphism $f_{\gamma}$ is Lipschitz with Lipschitz constant equal to that of $f_{n_{k+1}}$. Moreover:

(i) $\left.f_{\gamma}\right|_{\mathbb{R}^{n}-\stackrel{\circ}{X}_{\gamma}}=\mathrm{id}$.

(ii) For arbitrary $g \in G_{n_{k+1}}$, we have $f_{\gamma}\left(X_{\gamma g}\right)=X_{\gamma(g+1)}$, the diagram

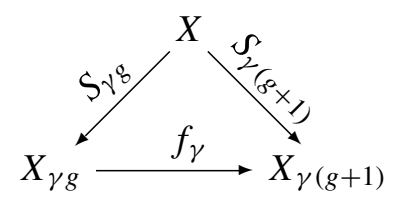

commutes, and $\left.f_{\gamma}\right|_{X_{\gamma g}}$ is an isometry. 
(iii) For arbitrary $\left(g_{k+1}, g_{k+2}, \ldots\right) \in G_{k+1}^{\infty}$,

$$
f_{\gamma}\left(X_{\left(\gamma, g_{k+1}, g_{k+2}, \ldots\right)}\right)=f_{\gamma}\left(X_{\left(\gamma, 1+g_{k+1}, g_{k+2}, \ldots\right)}\right) .
$$

Proof. This follows in a manner similar to [Malešič and Repovš 1999, Lemma 2]. Part (i) follows directly from condition (i) of Theorem 1. Part (ii) follows from condition (ii) of the same theorem. Finally, (ii) implies (iii).

Lemma 2. The homeomorphisms $h_{k}$ exhibit the following properties:

(i) $h_{k}^{-1}=\ell_{1} \circ \ell_{2} \circ \cdots \circ \ell_{k-1} \circ \ell_{k}$.

(ii) $h_{k}\left(X_{\alpha^{k}}\right)=X_{\beta^{k}}$ and $h_{k}\left(X_{\alpha^{k} \gamma}\right)=X_{\beta^{k} \gamma}$ for arbitrary multiindex $\gamma$.

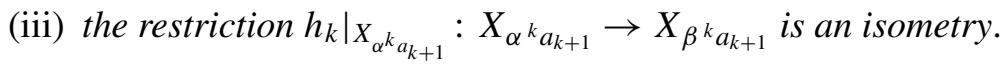

(iv) $\left.h_{k}\right|_{\mathbb{R}^{n}-\dot{X}_{\alpha^{k}}}=\left.h_{k+1}\right|_{\mathbb{R}^{n}-\dot{X}_{\alpha^{k}}}=\left.h_{k+2}\right|_{\mathbb{R}^{n}-\dot{X}_{\alpha^{k}}}=\cdots$.

Proof. This follows in a manner similar to [Malešič and Repovš 1999, Lemma 3]. Property (i) can be proved directly by examining the construction of $h_{k}$. Property (ii) follows from Lemma 1(ii)-(iii). Property (iii) holds since $\left.f_{\gamma}\right|_{X_{\gamma_{k+1}}}$ is an isometry. Property (iv) holds because of Lemma 1(i).

Lemma 3. $h_{k}$ and $h_{k^{-1}}$ are Lipschitz with equal Lipschitz constants for all values of $k$.

Proof. This requires the most modification of [Malešič and Repovš 1999] as multiple similarities with different constants of similarity are involved.

We fix the sequence $\alpha=\left(a_{1}, a_{2}, \ldots\right)$ of coordinates of the point $a \in|\mathbf{S}|$ and introduce the notion of depth of a point $x \in \mathbb{R}^{n}$ :

$$
\operatorname{dep} x=j \quad \text { if } x \in X_{\alpha^{j}}-{\stackrel{\circ}{\alpha^{j+1}}} .
$$

Additionally, let

$$
\operatorname{dep} x=0 \text { if } x \in X-{\stackrel{\circ}{a_{1}}}_{1} \text { and } \operatorname{dep} x=-1 \text { if } x \in \mathbb{R}^{n}-\stackrel{\circ}{X} .
$$

In the case $x \in \stackrel{\circ}{X}_{\alpha^{j}}$ for all $j \in \mathbb{N}$ (i.e. $x=a$ ) let $\operatorname{dep} x=\infty$.

For arbitrary distinct points $x, y \in \mathbb{R}^{n}$ we now estimate the expression $h_{k}(x)-$ $h_{k}(y)$. We may assume that $\operatorname{dep} x \leq \operatorname{dep} y$. As $x$ and $y$ are distinct, case dep $y=\infty$ and $\operatorname{dep} x=\infty$ is not possible.

Case 1 Let the Lipschitz constant of the homeomorphism $f_{i}$ be denoted by $\lambda_{i}$. Let $\lambda=\max \left\{\lambda_{i} ; 1 \leq i \leq M\right\}$ and $T=\max \left\{\left|G_{i}\right| ; 1 \leq i \leq M\right\}$, where $\left|G_{i}\right|$ denotes the number of elements of $G_{i}$. Hence the Lipschitz constants of the homeomorphisms $r_{1}, r_{2}, \ldots, \ell_{1}, \ell_{2}, \ldots$ do not exceed the number $\Lambda=\lambda^{T}$. Let $\operatorname{dep} y-\operatorname{dep} x \leq 1$, i.e.

$$
\operatorname{dep} x \in\{j, j+1\}, \operatorname{dep} y=j+1
$$


for some $j \in \mathbb{N} \cup\{-1,0\}$. By Lemma 2, (iii) and (iv), and because of the construction of $h_{k}$,

$$
\left|h_{k}(x)-h_{k}(y)\right|=\left|r_{j+1} \circ r_{j}(x)-r_{j+1} \circ r_{j}(y)\right| \leq \Lambda^{2}|x-y| .
$$

Case 2 Now let $\operatorname{dep} y-\operatorname{dep} x \geq 2$. First let the degrees be nonnegative, i.e.

$$
\operatorname{dep} x=j \geq 0 \text { and } \operatorname{dep} y \geq j+2
$$

for some $j \in \mathbb{N}$. (It may be dep $y=\infty$ as well.) Then

$$
x \in X_{\alpha^{j}}-\stackrel{\circ}{X}_{\alpha^{j+1}}, \quad y \in X_{\alpha^{j+2}} .
$$

For arbitrary disjoint compact sets $C_{1}, C_{2} \subset \mathbb{R}^{n}$, set

$$
\begin{gathered}
\mathrm{d}_{\min }\left(C_{1}, C_{2}\right)=\min \left\{|x-y| ; x \in C_{1}, y \in C_{2}\right\}, \\
\mathrm{d}_{\max }\left(C_{1}, C_{2}\right)=\max \left\{|x-y| ; x \in C_{1}, y \in C_{2}\right\} .
\end{gathered}
$$

The sets $X-\stackrel{\circ}{X}_{1}$ and $X_{2}$ are compact and disjoint; hence the numbers

$$
\mathrm{d}_{X}=\mathrm{d}_{\min }\left(X-\stackrel{\circ}{X}_{1}, X_{2}\right) \quad \text { and } \quad \mathrm{D}_{X}=\mathrm{d}_{\max }\left(X-\stackrel{\circ}{X}_{1}, X_{2}\right)
$$

exist. Since the similarity $S_{\alpha^{k}}$ maps the triple $\left(X, X_{a_{1}}, X_{\left(a_{1}, a_{2}\right)}\right)$ onto the triple $\left(X_{\alpha^{k}}, X_{\alpha^{k} a_{1}}, X_{\alpha^{k}\left(a_{1}, a_{2}\right)}\right)$, for each $k \in \mathbb{N}$, we have

$$
\frac{d_{\max }\left(X_{\alpha^{k}}-\stackrel{\circ}{X}_{\alpha^{k} a_{1}}, X_{\alpha^{k}\left(a_{1}, a_{2}\right)}\right)}{\mathrm{d}_{\min }\left(X_{\alpha^{k}}-\stackrel{\circ}{X}_{\alpha^{k} a_{1}}, X_{\alpha^{k}\left(a_{1}, a_{2}\right)}\right)} \leq \frac{\mathrm{D}_{X}}{\mathrm{~d}_{X}} .
$$

Hence $\left|h_{k}(x)-h_{k}(y)\right| \leq\left(\mathrm{D}_{X} / \mathrm{d}_{X}\right)|x-y|$.

Finally, let $\operatorname{dep} x=-1$ and dep $y \geq 1$, i.e., $x \in \mathbb{R}^{n}-\stackrel{\circ}{X}$ and $y \in X_{1}$. Then $h_{k}(x)=x$ and

$$
\frac{\left|h_{k}(x)-h_{k}(y)\right|}{|x-y|} \leq \frac{|x-y|+\left|y-h_{k}(y)\right|}{|x-y|} \leq 1+\frac{\operatorname{diam} X_{1}}{m},
$$

where $m=\inf \left\{|x-y| ; x \in \mathbb{R}^{n}-\stackrel{\circ}{X}, \quad y \in X_{1}\right\}$ (it is easy to show that $m>0$ ). To conclude, set

$$
L=\max \left\{\Lambda^{2}, \frac{\mathrm{D}_{X}}{\mathrm{~d}_{X}}, 1+\frac{\operatorname{diam} X_{1}}{m}\right\} .
$$

Then $\left|h_{k}(x)-h_{k}(y)\right| \leq L|x-y|$ for an arbitrary $k \in \mathbb{N}$ and $x, y \in \mathbb{R}^{n}$.

The estimate $\left|h_{k}^{-1}(x)-h_{k}^{-1}(y)\right| \leq L|x-y|$ can be proved analogously, using Lemma 2(i). 


\section{Main result}

Theorem 2. There exist uncountably many topologically distinct Lipschitz homogeneous wild Cantor sets in $\mathbb{R}^{3}$. In fact, these Cantor sets can all be constructed as simple Antoine Cantor sets with the same number of components of stage $n$ inside each component of stage $n-1$ and thus with the same Antoine graphs.

Proof. Use Theorem 1 with $M=2, G_{1}=Z_{60}$ and $G_{2}=Z_{60}$. Let $\mathbf{T}=\left(n_{1}, n_{2}, \ldots\right)$ be a fixed sequence of 1's and 2's. For each such sequence, construct a Lipschitz homogeneous Antoine Cantor set as in Theorem 1.

For $G_{1}$, let the similarities $S_{g}, g \in G_{1}$ be constructed so as to take the outer torus in Figure 1 to the smaller tori in the same figure. Each smaller torus in the chain is obtained from the previous one by rotating the large torus by $2 \pi / 60$ radians and then by rotating the small tori by $\pi / 2$ radians. The homeomorphism $f_{1}$ needed in Theorem 1 is constructed in a manner similar to that constructed in the example in [Malešič and Repovš 1999].

For $G_{2}$, let the similarities $S_{g}, g \in G_{2}$ be constructed so as to take the outer torus in Figure 2 to the smaller tori in Figure 2. Each smaller torus in the chain is obtained from the previous one by rotating the large torus by $2 \pi / 60$ radians and then by rotating the small tori by $\pi / 4$ radians. The homeomorphism $f_{2}$ needed in Theorem 1 is constructed in a manner similar to that constructed in the example in [Malešič and Repovš 1999]. The resulting Cantor set is Lipschitz homogeneously embedded by Theorem 1 .

Note that the Antoine graphs associated with any two Cantor set constructed in this way are the same.
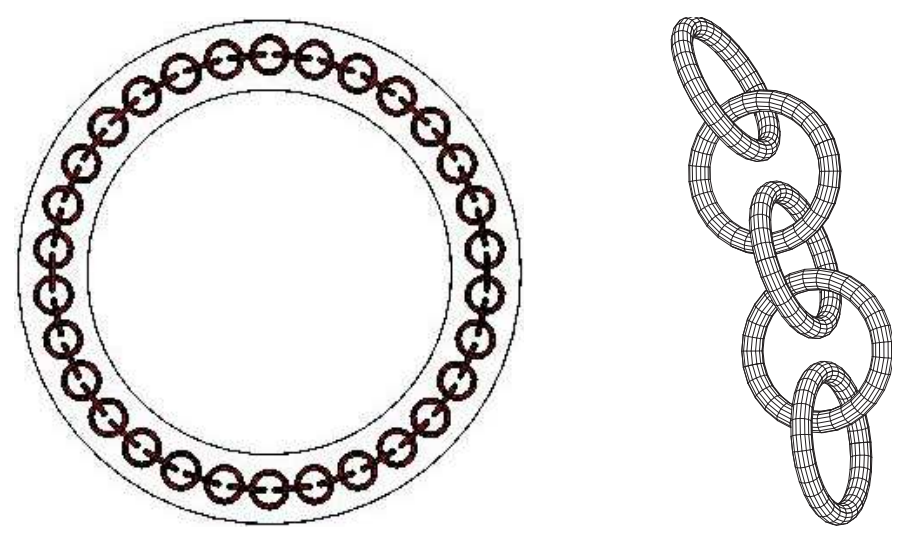

Figure 1. Left: A torus with 60 smaller similar tori linked in a simple chain inside, each of which is rotated by $\pi / 2$ radians from the previous one. Right: an enlargement of five of the smaller tori. 
Let $C_{1}$ be the Cantor set constructed as in Theorem 1 for one sequence of 1's and 2's and let $C_{2}$ be the Cantor set constructed using a different sequence. We need to show that these two Cantor sets are topologically inequivalently embedded. For this, using the result of Sher mentioned on page 289, it suffices to show that there is no homeomorphism of $\mathbb{R}^{3}$ to itself taking the large torus in Figure 1 to the large torus in Figure 2, and taking the chain of smaller tori in Figure 1 to the chain of smaller tori in Figure 2. If there were such a homeomorphism, the link formed by the centerlines of the small tori in Figure 1 would be topologically equivalent the link formed by the centerlines of the small tori in Figure 2. The next lemma shows that this is not the case.

There are uncountably many sequences of 1's and 2's. The argument above shows that each such sequence leads to a topologically distinct Lipschitz homogeneous wild Cantor set. This completes the proof of the theorem.

Lemma 4. The links formed by the center lines of the smaller tori in Figures 1 and 2 are inequivalent.

Proof. The link formed by the centerlines of the chain of smaller tori in Figure 2 is seen to be topologically embedded in $\mathbb{R}^{3}$ as follows. Starting at a fixed centerline and proceeding around the chain, the centerline can be twisted by a homeomorphism of $\mathbb{R}^{3}$ fixed outside of the large torus in such a way that all but one of the centerlines are embedded in the same manner as the link formed by the centerlines of the smaller tori in Figure 1. The remaining centerline in Figure 2 can be obtained from the corresponding centerline in Figure 1 by the following modification. One of the centerlines in Figure 1 is given 30 half twists before linking with the centerlines on either side. A computation of the version of the Jones polynomial introduced in [Kauffman 1988] shows that these links are topologically distinct,
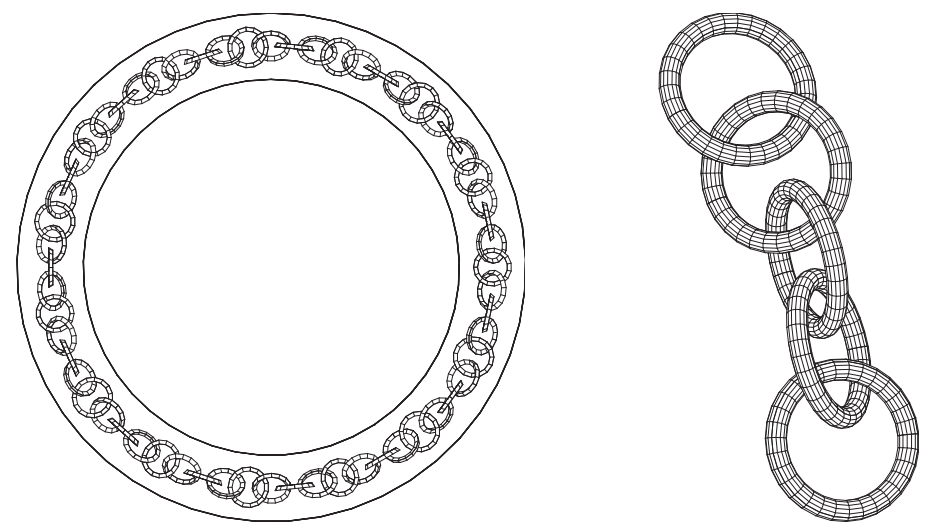

Figure 2. A similar chain, with each small torus rotated $\pi / 4$ relative to the previous one. 
and thus the Cantor sets are topologically distinct. In fact, all that is needed is to show that the span of the Kauffman polynomial (the highest power appearing in the polynomial minus the lowest power) is different in each case.

For details of a computation of the Kauffman polynomial in this setting, see [Garity $\geq 2006$ ]. For completeness, we outline the computation. We refer the reader to [Kauffman 1988] for details on the Kauffman bracket and Kauffman polynomial. Let $L$ represent an oriented link and $|L|$ represent a particular diagram for this link. The writhe of $L$ in the diagram $|L|$ is denoted by $\omega(|L|)$. Let $L_{+}, L_{-}$, and $L_{0}$ represented oriented link diagrams identical to $|L|$ except at one crossing, where $L_{+}$represents this crossing with positive crossing number, $L_{-}$with negative crossing number and $L_{0}$ with the crossing split and the orientation preserved. We use $X(L)$ to denote the Kauffman polynomial of $L$.

Open Chains. Let $C_{n}$ be a simple chain with $n$ links. An easy induction, or the multiplicativity of the Kauffman bracket relative to connected sums, shows that $\left\langle C_{n}\right\rangle=\left(-A^{4}-A^{-4}\right)^{n-1}$, where \langle\rangle is the Kauffman bracket. If we orient the links so the linking numbers alternate signs as in the superscripts below, we have

$$
\begin{aligned}
& X\left(C_{n}^{+-\cdots+-}\right)=X\left(C_{n}^{-+\cdots-+}\right)=\left(-A^{4}-A^{-4}\right)^{n-1} \text { for } n \text { odd, } \\
& X\left(C_{n}^{+-\cdots-+}\right)=(-A)^{-6}\left(-A^{4}-A^{-4}\right)^{n-1} \quad \text { for } n \text { even, } \\
& X\left(C_{n}^{-+\cdots+-}\right)=(-A)^{6}\left(-A^{4}-A^{-4}\right)^{n-1} \quad \text { for } n \text { even. }
\end{aligned}
$$

In each case, the span of the polynomial is $8^{n-1}$ and the maximum and minimum exponent in the polynomial can be read off. The maximum exponent is $4(n-1)$ if $n$ is odd and $4(n-1) \pm 6$ if $n$ is even. The minimum exponent is $-4(n-1)$ if $n$ is odd and $-4(n-1) \pm 6$ if $n$ is even.

Closed Chains. Now take a closed chain $L C_{2 n}$ with $2 n$ components and with no twists, as in Figure 1. Orient it so the linking numbers are alternately positive and negative. Consider three consecutive links and modify them as follows:

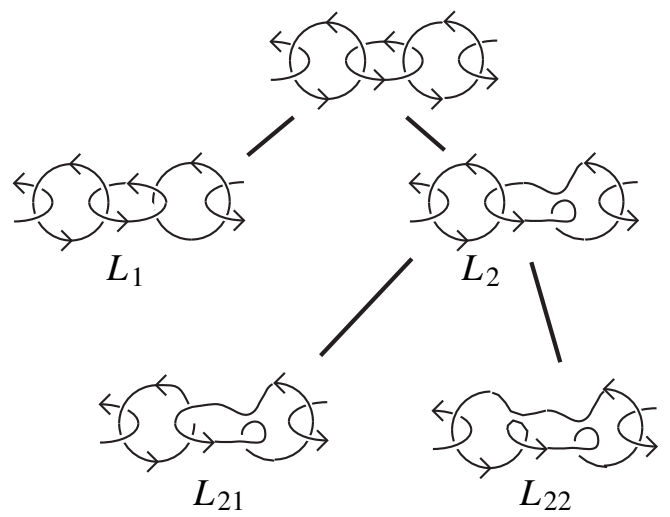


We now compute using the relation $A^{4} X(+)=A^{-4} X(-)+\left(A^{-2}-A^{2}\right) X(\rightrightarrows)$ and noting that the link labeled $L_{1}$ above is $C_{2 n}^{+\cdots+}$, the link $L_{21}$ is $C_{2 n-1}^{-\cdots+}$ and the link $L_{22}$ is $L C_{2 n-2}$ :

$$
\begin{aligned}
X\left(L C_{2 n}\right)= & A^{8} X\left(L_{1}\right)+A^{4}\left(A^{2}-A^{-2}\right) X\left(L_{2}\right) \\
= & A^{8} X\left(C_{2 n}^{+\cdots+}\right)+A^{4}\left(A^{2}-A^{-2}\right)\left(A^{-8} X\left(L_{21}\right)+A^{-4}\left(A^{-2}-A^{2}\right) X\left(L_{22}\right)\right) \\
= & A^{8} X\left(C_{2 n}^{+\cdots+}\right) \\
& \quad+A^{4}\left(A^{2}-A^{-2}\right)\left(A^{-8} X\left(C_{2 n-1}^{-\cdots+}\right)+A^{-4}\left(A^{-2}-A^{2}\right) X\left(L C_{2 n-2}\right)\right) \\
= & \cdots \\
= & \left(-A^{4}-A^{-4}\right)^{2 n-2}\left(-A^{6}-A^{-6}\right)-\left(A^{-2}-A^{2}\right)^{2} X\left(L C_{2 n-2}\right) .
\end{aligned}
$$

Thus

$$
X\left(L C_{2 n}\right)=\left(-A^{4}-A^{-4}\right)^{2 n-2}\left(-A^{6}-A^{-6}\right)-\left(A^{-2}-A^{2}\right)^{2} X\left(L C_{2 n-2}\right)
$$

This sets up a recursion relation that can be solved for $X\left(L C_{2 n}\right)$. The starting condition is that $X\left(L C_{2 \cdot 1}\right)=\left(-A^{2}-A^{-2}\right)$ has maximum exponent 2 and minimum exponent -2 , and hence span 4 .

The maximum exponent of $X\left(L C_{2 n}\right)$ is

$$
\max \left\{4(2 n-2)+6,4+\text { maximum exponent }\left(X\left(L C_{2 n-2}\right)\right)\right\}
$$

and the minimum exponent of $X\left(L C_{2 n}\right)$ is

$$
\min \left\{-4(2 n-2)-6 \text {, minimum exponent }\left(\left(L C_{2 n-2}\right)\right)-4\right\} \text {. }
$$

An easy induction now shows that $\operatorname{span}\left(X\left(L C_{2 \cdot n}\right)\right)=16(n-1)+12$ for $n \geq 2$.

Closed chains with twists. Take the case of a linked chain forming a loop with $2 n$ components, with $k$ positive half twists, where $k$ is even, in one of the links, $L C_{2 n}^{k}$.

Orient as in the previous case. By considering the diagram

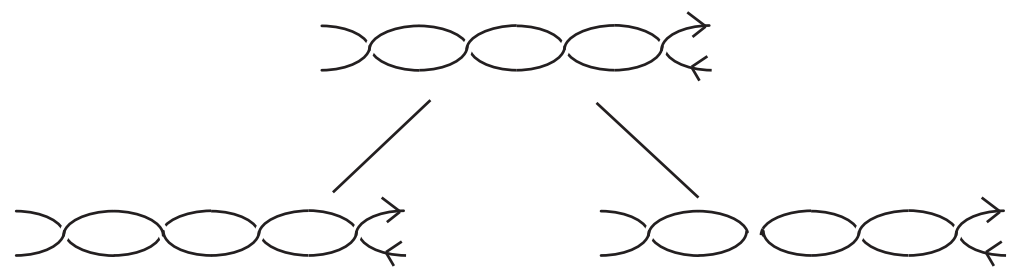

one can make the following computations.

$$
\begin{aligned}
& X\left(L C_{2 n}^{k}\right)=A^{-8} X\left(L C_{2 n}^{k-2}\right)+A^{-4}\left(A^{-2}-A^{2}\right) X\left(C_{2 n+1}\right), \\
& X\left(L C_{2 n}^{k}\right)=A^{-8} X\left(L C_{2 n}^{k-2}\right)+A^{-4}\left(A^{-2}-A^{2}\right)\left(-A^{-4}-A^{4}\right)^{2 n} .
\end{aligned}
$$

The starting point here is when $k=0, L C_{2 n}^{0}=L C_{2 n}$ 
The maximum exponent is $\max \left\{\max . \exp .\left(L C_{2 n}^{2 k-2}\right)-8,8 n-2\right\}$.

The minimum exponent is $\min \left\{\right.$ min. exp. $\left.\left(L C_{2 n}^{2 k-2}\right)-8,-8 n-6\right\}$.

An easy induction now shows that $\operatorname{span}\left(X\left(L C_{2 n}^{2 k}\right)\right)=16 n-4+4 k$, distinguishing topologically all the chains with $2 n$ links and different numbers of even twists. This completes the proof of the lemma.

\section{Other results and questions}

Using techniques similar to those used in the proof of Theorem 1, we can prove the following result. Note that in this case, we assume that $G$ is of the form $Z_{p} \times Z_{q}$ for some positive integers $p$ and $q$.

Theorem 3. For each $i, 1 \leq i \leq 2$, suppose that $f_{i}: \mathbb{R}^{n} \rightarrow \mathbb{R}^{n}$ is a Lipschitz homeomorphism and that:

(i) $\left.f_{i}\right|_{\mathbb{R}^{n}-\check{X}}=i d$;

(ii) $f_{1}\left(X_{(a, b)}\right)=X_{(a+1, b)}$ for $(a, b) \in G$;

(iii) $f_{2}\left(X_{(a, b)}\right)=X_{(a, b+1)}$ for $(a, b) \in G$ and the following diagrams commute:

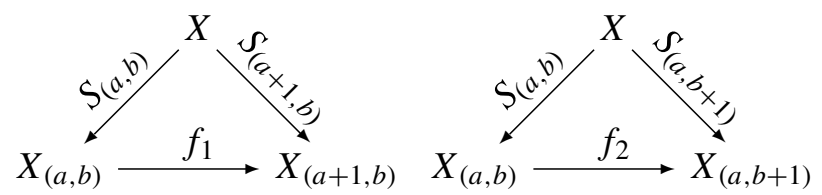

Then $|(\mathbf{S}, \mathbf{T})|$ is Lipschitz homogeneous in $\mathbb{R}^{n}$.

The construction suggested by the theorem is similar to the Blankinship construction [1951] for wild Cantor sets in $\mathbb{R}^{4}$.

Question. Can this theorem be used to show that a Lipschitz homogeneous wild Cantor set in $\mathbb{R}^{4}$ exists? This would require a more careful Blankinship-type construction, in which the successive stages in the construction were self-similar to the original stage.

\section{Acknowledgment}

We thank the referee for helpful suggestions.

\section{References}

[Antoine 1920] M. L. Antoine, "Sur la possibilité d'étendre l'homéomorphie de deux figures à leur voisinages”, C. R. Acad. Sci. Paris 171 (1920), 661-663. JFM 47.0524.01

[Blankinship 1951] W. A. Blankinship, "Generalization of a construction of Antoine", Ann. of Math. (2) 53 (1951), 276-297. MR 12,730c Zbl 0042.17601

[Eaton 1973] W. T. Eaton, "A generalization of the dog bone space to $E^{n ", ~ P r o c . ~ A m e r . ~ M a t h . ~ S o c . ~}$ 39 (1973), 379-387. MR 48 \#1238 Zbl 0262.57001 
[Garity $\geq 2006$ ] D. J. Garity, "Inequivalent Antoine Cantor sets with the same Antoine tree", in Proceedings of the Twentieth Annual Workshop in Geometric Topology (Park City, UT, 2003). To appear.

[Hutchinson 1981] J. E. Hutchinson, "Fractals and self-similarity", Indiana Univ. Math. J. 30:5 (1981), 713-747. MR 82h:49026 Zbl 0598.28011

[Kauffman 1988] L. H. Kauffman, "New invariants in the theory of knots", Amer. Math. Monthly 95:3 (1988), 195-242. MR 89d:57005 Zbl 0657.57001

[Malešič and Repovš 1999] J. Malešič and D. Repovš, "On characterization of Lipschitz manifolds", pp. 265-277 in New developments in differential geometry (Budapest, 1996), edited by J. Szenthe, Kluwer, Dordrecht, 1999. MR 99j:57042 Zbl 0939.57023

[Repovš et al. 1996] D. Repovš, A. B. Skopenkov, and E. V. Ščepin, " $C^{1}$-homogeneous compacta in $\mathbb{R}^{n}$ are $C^{1}$-submanifolds of $\mathbb{R}^{n}$ ", Proc. Amer. Math. Soc. 124:4 (1996), 1219-1226. MR 1301046 (97f:58008) Zbl 0863.53004

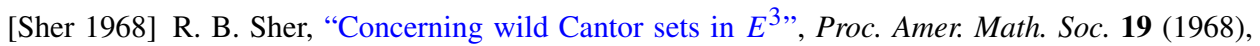
1195-1200. MR 38 \#2755 Zbl 0165.57202

[Wright 1986] D. G. Wright, "Rigid sets in $E^{n ", ~ P a c i f i c ~ J . ~ M a t h . ~ 121: 1 ~(1986), ~ 245-256 . ~ M R ~ 87 b: ~}$ 57011 Zbl 0697.12018 Zbl 0586.57009

Received April 13, 2004.

\section{DENNIS GARITY}

MATHEMATICS DEPARTMENT

OREGON STATE UNIVERSITY

CORVALLIS, OR 97331

UNITED STATES

garity@math.oregonstate.edu

http://www.math.oregonstate.edu / garity

DUŠAN REPOVŠ

Institute of Mathematics, Physics And Mechanics

UNIVERSITY OF LJUBLJANA

JADRANSKA 19, P.O.BOX 2964

LJUBLJANA

SLOVENIA

dusan.repovs@guest.arnes.si

MATJAŽ ŽELJKO

Institute of Mathematics, Physics And Mechanics

UNIVERSITY OF LJUBLJANA

JADRANSKA 19, P.O.BOX 2964

LJUBLJANA

SLOVENIA

matjaz.zeljko@fmf.uni-lj.si 